\title{
Salivary cortisol, cortisone and serum cortisol concentrations are related to age in healthy children and young people
}

\author{
Salivary cortisol and cortisone in childhood \\ Andrew Titman, ${ }^{1}$ Victoria Price, ${ }^{2}$ Daniel Hawcutt, ${ }^{2}$ Chris Chesters, ${ }^{3}$ Murtaza Ali, ${ }^{4}$ Gianluca \\ Cacace, ${ }^{4}$ Gill A Lancaster, ${ }^{5}$ Matthew Peak, ${ }^{6}$ Joanne C Blair ${ }^{7}$ \\ Department of Mathematics and Statistics, Lancaster University, ${ }^{1}$ NIHR Clinical Research \\ Facility, Alder Hey Children's Hospital, ${ }^{2}$ Department of Biochemistry, Alder Hey \\ Children's Hospital, ${ }^{3}$ University of Liverpool School of Medicine, ${ }^{4}$ School of Primary, \\ Social and Community Care \& Keele Clinical Trials Unit, Keele University ${ }^{5}$ Clinical \\ Research Division and ${ }^{6}$ Department of Endocrinology, Alder Hey Children's Hospital ${ }^{7}$
}

Acknowledgements: We are very grateful to Dr Jo Adaway and Mrs Sherly George who oversaw the salivary cortisol and cortisone assays, which were undertaken in the Department of Clinical Biochemistry, Wythenshawe Hospital, Manchester University NHS Foundation Trust.

\section{Summary}

Background: Saliva is an ideal medium in which to measure cortisol in children. However, there are very few data reporting salivary cortisol or cortisone concentrations in healthy children since the introduction of liquid chromatography mass spectrometry (LC MS/MS) to routine laboratory practice.

Design: Early morning serum cortisol, salivary cortisol and cortisone were measured on fasting samples, and salivary hormones were measured in samples collected every 2 hours during waking hours, and 30 minutes after waking the following morning.

Participants: 43 healthy paediatric volunteers (19 female), median age 11.5 years, range 6.3 - 18.7, participated.

Results: Early morning serum cortisol $(265 \mathrm{nmol} / \mathrm{L}, 156$ - 516) correlated strongly with salivary cortisol (4.7nmol/L, 1.1-14.6) and cortisone (28.8nmol/L, 11.7-56.6), $p<0.0001$ for both. Serum cortisol, salivary cortisol and salivary cortisone correlated directly with age $(p<0.0001, p=0.002$ and $p=0.015$ respectively), and salivary cortisone / cortisol ratio correlated indirectly with age ( $p=0.007)$. Between 08.00 and 21.00 , area under the curve for salivary cortisol (mean $\pm 1 \mathrm{SD}$ ) was $41.8 \pm 19.1$ and for cortisone, $213.0 \pm 61.2$. Salivary cortisol was undetectable in 25/130 (19\%) of samples collected after 13.00 while cortisone was always detectable.

Discussion: Salivary cortisol and cortisone concentrations are strongly related to serum cortisol concentrations, however cortisone may be a preferable measure as cortisol is often undetectable. All measures of cortisol secretion correlated directly with age, suggesting age 
related reference ranges may reduce the risk of overdiagnosis of adrenal insufficiency in the young.

Key words: Child, saliva, serum, cortisol, cortisone 


\section{Background}

The utility of saliva as a medium in which to measure hormones has been recognised for more than fifty years. More recently, developments in sample analysis have generated renewed interest in the use of saliva as a tool for the diagnosis of conditions of cortisol excess $^{1}$ and deficiency, ${ }^{2}$ adequacy of hydrocortisone replacement therapy ${ }^{3}$ and as a tool in psychology and sport research.

Cortisol activity is regulated at a tissue level by two isoforms of the enzyme $11 \beta$ hydroxysteroid dehydrogenase (11ßHSD). Cortisol is inactivated by conversion to cortisone

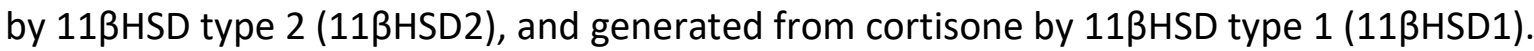
Cortisol and aldosterone show equal affinity for the mineralocorticoid receptor in vitro, ${ }^{4}$ and inactivation of cortisol to cortisone by $11 \beta \mathrm{HSD} 2$ in mineralocorticoid responsive tissues protects the receptor from cortisol mediated activation. Measurement of the ratio of cortisol / cortisone in serum has been identified as a potential tool for screening for abnormalities of $11 \beta \mathrm{HSD} 2$ activity. ${ }^{5}$ Age related changes in $11 \beta \mathrm{HSD} 2$ activity have also been implicated in the aetiology of age related hypertension. ${ }^{6}$

Ninety to $95 \%$ of serum cortisol circulates bound to cortisol binding globulin and generic binding proteins and only $5-10 \%$ is unbound or 'free' and biologically active. Free, but not bound, cortisol can diffuse through the parotid where it is converted rapidly to cortisone, and into saliva. The ratio of cortisone / cortisol is approximately 0.25 in the serum of heathy young adults. In saliva, this ratio is reversed to approximately six. ${ }^{7}$

The use of saliva as a medium to measure cortisol in children has a number of advantages over serum. Only free, biologically active hormone is measured. Samples can be collected at home, school and during normal daily activities, and artificial increases in cortisol due to stress induced by hospital visits and blood tests are avoided. Furthermore, disruption to the family is reduced with fewer lost days from school and work. There are also potential healthcare cost savings.

To our knowledge, there are few data describing salivary cortisone concentrations in healthy children. An increase in the ratio of salivary cortisol/cortisone during the first year of life, followed by stability throughout childhood and in to young adult life has been described, while salivary cortisol concentrations have variably been associated with sex, pubertal development and body mass index. ${ }^{8-15}$

Adult studies have reported that salivary cortisone is a superior marker of serum cortisol than salivary cortisol, that salivary cortisol may be undetectable in healthy young men when serum cortisol concentrations fall below $74 \mathrm{nmol} / \mathrm{L}$ while salivary cortisone remains detectable, and that the use of salivary cortisone allows monitoring of hydrocortisone therapy without the risk of artificially elevated salivary cortisol concentrations due to saliva contamination with hydrocortisone. ${ }^{7}$ 
It was the purpose of this study to determine, in the paediatric age group, which salivary biomarker correlated most strongly with serum cortisol, whether either was undetectable during waking hours, and whether age and gender should be considered in the design of a study to generate reference data for use in clinical practice.

\section{Subjects and Methods}

\section{$\underline{\text { Subjects }}$}

Information about the study was shared with parents of children attending outpatient clinics at Alder Hey Children's Hospital, Liverpool. Parents were invited to discuss participation in the study with their healthy sons and daughters at home, supported by age appropriate written information.

Participants were eligible to participate in the study if neither they, nor their parents had any concerns about their health, if they were not attending any medical appointments relating to their health and if they were not taking any medications. The following were specifically excluded: conditions likely to affect serum cortisol or CBG including abnormalities of thyroid or anterior pituitary hormone secretion, psychiatric pathology, type I or type 2 diabetes, cystic fibrosis, protein losing enteropathies, nephrotic syndrome and patients undergoing renal dialysis. Children with a family history of adrenal insufficiency due to an inherited condition, including congenital adrenal hyperplasia or oral conditions likely to result in blood contamination of saliva samples were also excluded.

Children below the age of five years were excluded because of concerns that the cotton wool roll used to collect saliva in the Salivette device may present a choking hazard.

\section{Study protocol}

Participants attended the Clinical Research Facility, Alder Hey Children's Hospital at 08.00 in the fasted state, and before brushing their teeth. Written informed consent and, where appropriate, assent was obtained for all study participants.

A blood sample was collected on arrival, or following the application of local anaesthetic if requested. Participants rinsed their mouth with tap water and collected a saliva sample using the Salivette sampling device (Sarstedt, Rommelsdorf, Germany).

Patients were weighed on electronic scales and measured using a wall mounted stadiometer. Height and body mass index standard deviations were derived from normative data reported by the World Health Organisation Multicentre Growth Reference Study Group. ${ }^{16}$ Participants were then given breakfast and discharged from the Clinical Research Facility. 
A subgroup of participants agreed to collect a saliva sample for every two hours for the rest of the day, until they went to bed at the normal time. Patients did not eat, drink or brush their teeth in the hour before collecting a saliva sample. A final saliva sample was collected in the fasting state 30 minutes after waking, and before participants had cleaned their teeth, to capture the early morning cortisol awakening response.

\section{$\underline{\text { Assays }}$}

Serum cortisol was measured in the clinical laboratory at Alder Hey Children's Hospital by immunoassay using the Siemens Immulite 2000XPi immunoassay system (Siemens Diagnostics, Camberley, Surrey), an automated immunoassay analyser using reagents supplied by the manufacturer. This assay has an intra- and inter-assay coefficient of variation of $<5 \%$ and $<7 \%$ respectively.

LC-MS/MS analysis for salivary cortisone and cortisol was performed using a Waters Xevo TQS micro mass spectrometer and a Waters Acquity iclass LC system with an electrospray source operated in positive ionization mode following sample preparation by protein precipitation. The LOQ for both analytes is $0.3 \mathrm{nmol} / \mathrm{L}$, with intraassay $\mathrm{CV}$ of $<=3.3 \mathrm{nmol} / \mathrm{L}$ for cortisol, $<=3.2 \mathrm{nmol} / \mathrm{L}$ for cortisone and interassay $\mathrm{CV}$ of $<=4.5 \%$ for cortisol and $<=3 \%$ for cortisone at concentrations 5-150 nmol/L.

\section{Statistical analysis}

Simple associations between pairs of variables were assessed using Pearson's correlation coefficient. Linear regression was used to assess the association between a response variable and two or more variables. Since the distribution of the response variables (salivary cortisol, salivary cortisone, serum cortisol) was positively skewed, regression was performed using log-transformed versions of the variables.

Based on the regression analysis, pointwise $95 \%$ prediction intervals for serum cortisol, salivary cortisol and the salivary cortisone/cortisol ratio were constructed based on the critical values of the relevant t-distribution using the estimated residual standard deviation and back-transforming to the natural scale.

The longitudinal measurements of salivary cortisol and cortisone were analysed by using linear mixed models, ${ }^{17}$ again working with log-transformed variables, and assuming correlated random intercepts for log-cortisol and log-cortisone and assuming the residual errors at each measurement time are correlated. In cases where the measurement fell below the detectable limits, a value equal to the limit of detection was imputed. If the time of first measurement was not recorded, a value of 8am was imputed.

The models assumed separate log-linear trends in salivary cortisol and salivary cortisone over time, from which the trend in the cortisone/cortisol ratio was also inferred. The models were fitted using the $n / m e$ package within R. ${ }^{18}$ 


\section{Ethics}

The study was approved by the London - City Road \& Hampstead Research Ethics Committee, reference 15/LO/1840.

\section{Results}

\section{$\underline{\text { Patient characteristics }}$}

Forty three participants took part in the study. Median age was 11.5 years (range $6.3-18.7$ ) and 19 were female. Median height was 0.25 SD (-2.5 to 2.5), and BMI SDS 0.2 (-2.5 to 3.5).

A subgroup of 29 participants, median age 12.2 years, (6.4 to 18.4), collected saliva samples throughout the day, of whom 26 , median age 11.4 years $(6.4-16.4), 11$ females, also collected a sample 30 minutes after waking.

Early morning paired measurements of serum cortisol, salivary cortisol and salivary cortisone

Early morning measurements of serum and salivary hormones and ratio of salivary cortisone / cortisol, presented for the whole population and by gender, are given in Table 1.

Salivary cortisol correlated strongly to serum cortisol (rho $=0.77, p<0.0001$ ). The correlation between salivary cortisone and serum cortisol was weaker ( $r$ o $=0.69, p<0.0001$ ). The correlation between serum cortisol and salivary biomarkers was not strengthened by using the sum of salivary cortisol + salivary cortisone ( $R^{2} 0.603$ vs $\left.0.594, p=0.348\right)$.

Serum cortisol was related to age, with an increase in serum cortisol of $17 \mathrm{nmol} / \mathrm{L}$ per year of age $(p<0.0001)$. Age accounted for approximately $32 \%$ of the between patient difference in serum cortisol $\left(R^{2}=0.315\right)$. The relationship between serum cortisol and age is illustrated in Figure $1 \mathrm{~A}$.

Salivary cortisol also correlated strongly with age, with an increase of $11 \%$ for each year of age $(p=0.002)$. Age accounted for approximately $20 \%$ of the between individual variation in measurements $\left(R^{2}=0.201\right)$. The relationship between salivary cortisol and age is illustrated in Figure 1B.

There was a weaker, though still statistically significant, association between salivary cortisone and age, with an increase of $5 \%$ for each year of age $(p=0.015)$. Age accounted for approximately $14 \%$ of the between individual variation in measurements $\left(R^{2}=0.140\right)$. The ratio of cortisone / cortisol ratio showed an inverse relationship with age, decreasing by $5.3 \%$ for every year of age $(p=0.007)$. Age accounted for $15 \%$ of the variability between individuals $\left(R^{2}=0.146\right)$. The relationship between salivary cortisone / cortisol ratio and age is illustrated in Figure 1D. 
There was no difference between males and females in serum cortisol, salivary cortisol, salivary cortisone or salivary cortisone / cortisol ratio. Similarly, no significant associations were found between BMI Z-score (standardized difference between the child's BMI and the average for their age and gender) and any of the response variables.

\section{Cortisol Awakening Response}

The median salivary cortisol concentration was $9.7 \mathrm{nmol} / \mathrm{L}(3.9-19.9)$, salivary cortisone $38.2 \mathrm{nmol} / \mathrm{L}(19.5-55.3)$, and ratio of cortisone / cortisol $4.0(2.1-5.8)$.

\section{$\underline{\text { Saliva cortisol and cortisone profiles during waking hours }}$}

In total, 190 samples were contributed from 29 participants.

For samples collected between 08.00 and 21.00 , the area under the curve (mean \pm 1 SD) for salivary cortisol was $41.8 \pm 19.1$ and for salivary cortisone $213.0 \pm 61.2$.

Linear mixed effects modelling, adjusting for age or BMI_z, indicate that during waking hours, salivary cortisol concentrations fell on average by $17 \%$ per hour ( $95 \% \mathrm{Cl}: 14 \%-19 \%)$ and salivary cortisone by $13 \%$ (95\% Cl: $11 \%-15 \%)$, resulting in a decreasing trend in cortisone / cortisol ratio (Table 2). A one standard deviation increase in BMI z-score was associated with a $23 \%(95 \% \mathrm{Cl}: 4 \%-48 \%)$ increase in cortisol throughout the day. There was a non-significant effect of BMI z-score on cortisone levels. The effect of age on cortisol and cortisone levels was weaker than observed in the early morning sample and non-significant for salivary cortisol.

The variance components estimates indicate $22 \%$ of the residual variability in log-cortisol measurements and $8 \%$ of the variability in log-cortisone measurements was explainable through inter-person variability.

Cortisol was below detectable limits in 25 samples, of which one was collected at 13.00, two were collected at 15.00, four were collected at 17.00, four were collected at 19.00 and 14 were collected at 21.00. Salivary cortisone was detectable in all samples.

\section{Discussion}

To our knowledge this is the first study to describe salivary cortisone profiles, the relationship between salivary cortisone and serum cortisol, and salivary cortisone / cortisol ratios in healthy children and young people. In samples collected in the early morning, salivary cortisol correlated more strongly with serum cortisol than salivary cortisone, and combining measurements of salivary cortisone and salivary cortisol did not strengthen the relationship between salivary biomarkers and serum cortisol. However, measurement of salivary cortisone is likely to be required in samples collected later in the day, as salivary 
cortisol was below detectable limits in $13 \%$ of saliva samples, including $19 \%$ of samples collected after 13.00 . In contrast, salivary cortisone was detectable in all samples.

Our observations contrast to studies in healthy young adult male volunteers, in whom salivary cortisone was a superior marker of serum cortisol than salivary cortisol. ${ }^{3}$ The discrepancy in these paediatric and adult data may reflect the wider range of serum cortisol concentrations over which this association was studied in the adult cohort. Salivary cortisol was below the level of detection in these adult studies when serum cortisol fell below $74 \pm$ $29 \mathrm{nmol} / \mathrm{L}$, while salivary cortisone remained detectable.

The ratio of salivary cortisol / cortisone demonstrated marked inter-individual variability, and was related to age but not gender. For the cohort as a whole however, the ratio measured in the early morning sample was similar to that reported in healthy adults [paediatric population 5.9 (2.2 - 14.4), adult population $6.4 \mathrm{nmol} / \mathrm{L}(2.4-14.6)$ ]. ${ }^{7}$ A circadian rhythm in the ratio of salivary cortisone / cortisol has been reported in adult volunteers, ${ }^{7}$ and was observed in our cohort too. If this ratio is to be used to screen populations for abnormalities in the activity of $11 \beta \mathrm{HSD}$, it may be important to standardise the time of day at which samples are collected.

The ratio of cortisone / cortisol has been implicated in the regulation of blood pressure in adult life. It may be that age-related changes in cortisol concentrations and cortisone / cortisol ratios contribute to the physiological rise in blood pressure observed during childhood. Lower cortisol concentrations and more rapid cortisol conversion to cortisone in the younger children would protect the mineralocorticoid receptor from cortisol mediated activation.

In our population we could account for $15 \%$ of the variability in cortisone / cortisol ratio by age, with no effect of gender. These data suggest considerable genetic heterogeneity in the activity of the two enzymes that regulate this ratio. Such genetic variability in the regulation of cortisol and cortisone may contribute to the risk of developing hypertension, and may influence the pharmacokinetics of hydrocortisone and clinical outcomes for patients with adrenal insufficiency treated with this medication.

Beyond the first year of life, age is not generally considered to be an important factor in the interpretation of tests of adrenal reserve. In our previous work, studying adrenal reserve in children with asthma treated with inhaled corticosteroids, early morning serum cortisol increased by $11 \mathrm{nmol} / \mathrm{L}$ per year of age, ${ }^{19}$ slightly less than the increase of $17 \mathrm{nmol} / \mathrm{L}$ per year of age that we observed in this cohort of healthy children and young people.

We took a pragmatic approach to the design of this study, and as most clinicians are used to the concept of an early morning cortisol measurement being one collected between 08.00 and 09.00, rather than one timed to waking, we elected to adopt this time point for our study. However, it is very likely that the adolescent patients woke at a later time than the 
younger patients, and the effect of the cortisol awakening response on the concentration of cortisol in the serum sample and associated saliva sample is likely to be greater in older than in younger children. This may account for the higher hormone concentrations observed in older children.

Previous studies of serum cortisol in much larger cohorts of healthy children, reported up to fivefold differences in mean cortisol measured over 24 hours, with no effect of age or gender. ${ }^{20}$ The area under the curve of salivary cortisol concentrations measured during waking hours in our cohort also showed approximately a fivefold difference between those with the lowest and those with the highest cortisol concentrations. The wide range of measurements in the baseline, early morning saliva sample is likely to be influenced by individual stress responses to attendance at hospital and a blood test. Variability of cortisol and cortisone concentrations in saliva samples collected at other times is also likely to be influenced by environmental factors, and illustrates the difficulty of defining 'normal' and 'abnormal' cortisol concentrations within populations. It is also likely that genetic variability in a spectrum of genes that regulate cortisol metabolism, transport and sensitivity influence serum and salivary hormone concentrations, and that environmental and genetic factors interact in a complex manner. ${ }^{21}$

In contrast to previous authors, ${ }^{9}$ in this study and in our previous work in children with asthma, we found no relationship between gender and early morning serum cortisol concentrations. An association between stage of puberty and salivary cortisol ${ }^{13}$ has also been reported. A meta-analysis of studies reporting salivary cortisol in healthy children reported that salivary cortisol concentrations were higher in boys during early and mid childhood, and were lower than girls from the age of eight years. ${ }^{22}$ It may be that our study population was too small to identify gender related differences.

In conclusion, in this cohort of healthy children and young people serum cortisol concentration, salivary cortisol and cortisone concentrations, and the ratio of salivary cortisone / cortisol are related to age, but not gender. Circadian changes in the salivary cortisone / cortisol ratio observed previously in adults were also observed in this cohort. Inter-individual variability in all measures was considerable. Our data suggest that interpretation of cortisol concentrations should consider age and clinical profile to avoid over diagnosis of adrenal insufficiency in the youngest children. Individual variability in the cortisone / cortisol ratio is likely to reflect differences in the activity of 11ßHSD1 and 2 and the significance of these observations for the regulation of blood pressure and hydrocortisone metabolism deserve further investigation.

The data reported in this study reveals interesting trends in salivary biomarkers with age. We now intend to extend the dataset to prepare robust reference data for salivary cortisol and cortisone in healthy children and young people for use in clinical practice. 


\section{Funding}

This study was funded by the Endocrine Research Fund, Alder Hey Charity

\section{Acknowledgements}

We are very grateful to Dr Jo Adaway and Mrs Sherly George who oversaw the salivary cortisol and cortisone assays, which were undertaken in the Department of Clinical Biochemistry, Manchester University NHS Foundation Trust.

\section{Data Sharing}

The data that support the findings of this study are available from the corresponding author upon reasonable request.

\section{Conflict of Interest Statement}

All authors declare they have no conflict of interest.

\section{References}

1. Nieman LK, Biller BMK, Findling, JW, et al, The Diagnosis of Cushing's Syndrome: An Endocrine Society Clinical Practice Guideline J Clin Endocrinol Metab ; 93, (5): 1526-1540

2. Perogamvros I, Keevil BG, Ray DW, Trainer PJ. Salivary cortisone is a potential biomarker for serum free cortisol. J Clin Endocrinol Metab. 2010 Nov;95(11):4951-8.

3. Ceccato F, Albiger N, Reimondo G, et al. Assessment of glucocorticoid therapy with salivary cortisol in secondary adrenal insufficiency. Eur J Endocrinol. 2012 Dec;167(6):769-76.

4. Arriza JL, Weinberger C, Cerelli G, et al. Cloning of human mineralocorticoid receptor complementary DNA: structural and functional kinship with the glucocorticoid receptor. Science. 1987 Jul 17;237(4812):268-75. 
5. Carvajal CA, Tapia-Castillo A, Valdivia CP, et al. Serum Cortisol and Cortisone as Potential Biomarkers of Partial 11 $\beta$-Hydroxysteroid Dehydrogenase Type 2 Deficiency. Am J Hypertens. 2018 Jul 16;31(8):910-918

6. Campino C, Martinez-Aguayo A, Baudrand R, et al. Age-related changes in $11 \beta-$ hydroxysteroid dehydrogenase type 2 activity in normotensive subjects. Am J Hypertens. 2013 Apr;26(4):481-7.

7. Debono M, Harrison RF, Whitaker MJ, et al. Salivary Cortisone Reflects Cortisol Exposure Under Physiological Conditions and After Hydrocortisone. J Clin Endocrinol Metab. 2016 Apr;101(4):1469-77.

8. Dötsch J, Hohenberger I, Peter M, Sippell W, Dörr HG. Evidence for change of 11 beta-hydroxysteroid dehydrogenase activity during infancy and childhood. Pediatr Res. 2000 Nov;48(5):697-700.

9. Netherton C, Goodyer I, Tamplin A, Herbert J. Salivary cortisol and dehydroepiandrosterone in relation to puberty and gender. Psychoneuroendocrinology. 2004 Feb;29(2):125-40.

10. Kang JY, Park JY, Chun SI, Suh HS, Lee K, Ahn RS. Puberty-related changes in cortisol, dehydroepiandrosterone, and estradiol-17 $\beta$ secretions within the first hour after waking in premenarcheal girls. Neuroendocrinology. 2014;99(34):168-77

11. Matchock RL, Dorn LD, Susman EJ. Diurnal and seasonal cortisol, testosterone, and DHEA rhythms in boys and girls during puberty. Chronobiol Int. 2007;24(5):969-90.

12. Oskis A, Loveday C, Hucklebridge F, Thorn L, Clow A. Diurnal patterns of salivary cortisol across the adolescent period in healthy females.

Psychoneuroendocrinology. 2009 Apr;34(3):307-16.

13. Gunnar, M.R., Wewerka, S., Frenn, K. et al. (2009) Developmental changes in hypothalamus-pituitary-adrenal activity over the transition to adolescence: normative changes and associations with puberty. Development and Psychopathology, 21, 69-85.

14. Shirtcliff, E.A., Allison, A.L., Armstrong, J.M. et al. (2012) Longitudinal stability and developmental properties of salivary cortisol levels and circadian rhythms from childhood to adolescence. Developmental Psychobiology, 54, 493-502.

15. Törnhage $\mathrm{C}$. Reference values for morning salivary cortisol concentrations in healthy school-aged children.J Pediatr Endocrinol Metab. 2002 Feb;15(2):197204.

16. WHO Multicentre Growth Reference Study Group. WHO Child Growth Standards based on length/height, weight and age. Acta Paediatr Suppl. 2006 Apr;450:7685.

17. Mixed-Effects Models in S and S-PLUS, Jose C. Pinheiro and Douglas M. Bates. Springer-Verlag, New York, 2000. 
18. Linear and Nonlinear Mixed Effects Models, Jose Pinheiro and Douglas Bates and Saikat DebRoy and Deepayan Sarkar and R Core Team. 2019, R package version 3.1-140.

19. Blair J, Lancaster G, Titman A, et al. Early morning salivary cortisol and cortisone, and adrenal responses to a simplified low-dose short Synacthen test in children with asthma. Clin Endocrinol. 2014;80(3):376-83.

20. Knutsson U, Dahlgren J, Marcus C, et al Circadian cortisol rhythms in healthy boys and girls: relationship with age, growth, body composition, and pubertal development. J Clin Endocrinol Metab. 1997 Feb;82(2):536-40.

21. Van Hulle CA, Shirtcliff EA, Lemery-Chalfant K, Goldsmith HH. Genetic and environmental influences on individual differences in cortisol level and circadian rhythm in middle childhood. Horm Behav. 2012 Jun;62(1):36-42

22. van der Voorn B, Hollanders JJ, Ket JCF, Rotteveel J, Finken MJJ.

23. Gender-specific differences in hypothalamus-pituitary-adrenal axis activity during childhood: a systematic review and meta-analysis. Biol Sex Differ. 2017 Jan 19;8:3 
Table 1: Early morning serum cortisol, salivary cortisol and salivary cortisone in healthy children and young people

\begin{tabular}{|l|c|c|c|}
\hline & Males (N=22) & Females (N=20) & Entire cohort \\
\hline $\begin{array}{l}\text { Serum cortisol } \\
\text { (nmol/L) }\end{array}$ & $250(164-516)$ & $302(156-419)$ & $265(156-516)$ \\
\hline $\begin{array}{l}\text { Salivary cortisol } \\
\text { (nmol/L) }\end{array}$ & $4.1(1.72-14.4)$ & $5.6(1.1-14.6)$ & $4.7(1.1-14.6)$ \\
\hline $\begin{array}{l}\text { Salivary cortisone } \\
\text { (nmol/L) }\end{array}$ & $27.2(11.7-45.4)$ & $29.7(56.6-14.7)$ & $28.8(11.7-56.6)$ \\
\hline $\begin{array}{l}\text { Ratio of salivary } \\
\text { cortisone / cortisol }\end{array}$ & $5.9(2.2-9.8)$ & $5.8(3.6-14.4)$ & $5.9(2.2-14.4)$ \\
\hline
\end{tabular}


Table 2: Fixed effect parameters for a linear mixed model for log-cortisol and log-cortisone profiles during waking hours.

\begin{tabular}{|c|c|c|c|}
\hline & Parameter & Est & $95 \% \mathrm{Cl}$ \\
\hline \multirow{4}{*}{$\begin{array}{l}\overline{0} \\
\stackrel{\mathscr{H}}{0} \\
0\end{array}$} & Intercept & 1.70 & $(0.77,2.63)$ \\
\hline & Time (hrs) & -0.18 & $(-0.21,-0.15)$ \\
\hline & BMI z-score & 0.21 & $(0.04,0.39)$ \\
\hline & Age & 0.02 & $(-0.05,0.10)$ \\
\hline \multirow{4}{*}{ 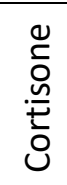 } & Intercept & 3.06 & $(2.62,3.51)$ \\
\hline & Time (hrs) & -0.14 & $(-0.16,-0.12)$ \\
\hline & BMI z-score & 0.05 & $(-0.03,0.13)$ \\
\hline & Age & 0.05 & $(0.01,0.08)$ \\
\hline
\end{tabular}


Figure 1: Serum cortisol (A), salivary cortisol (B), salivary cortisone (C) and salivary cortisone / cortisol ratio (D) with pointwise $95 \%$ prediction intervals in relation to age in healthy children and young people. Serum and saliva concentrations are shown as nmol/L.

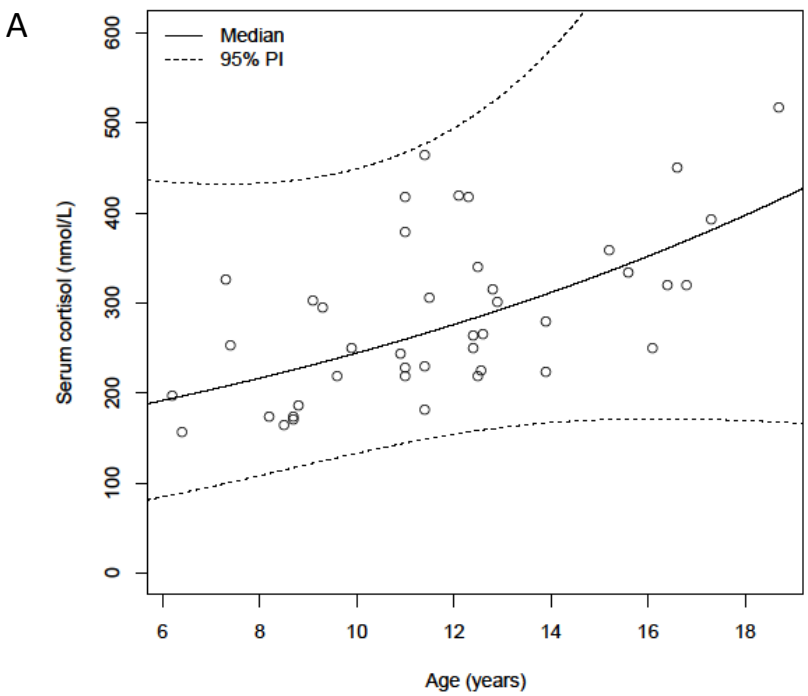

B

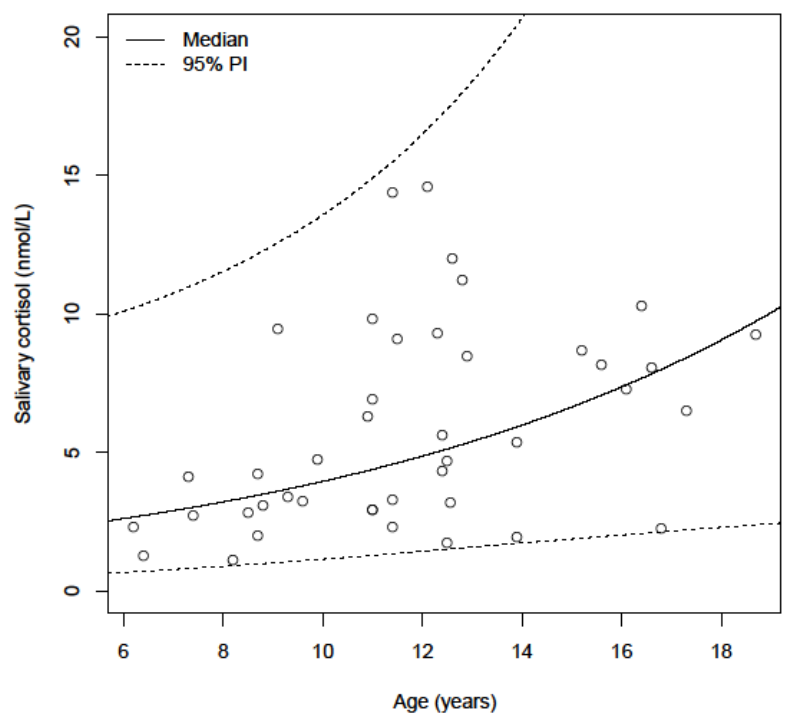



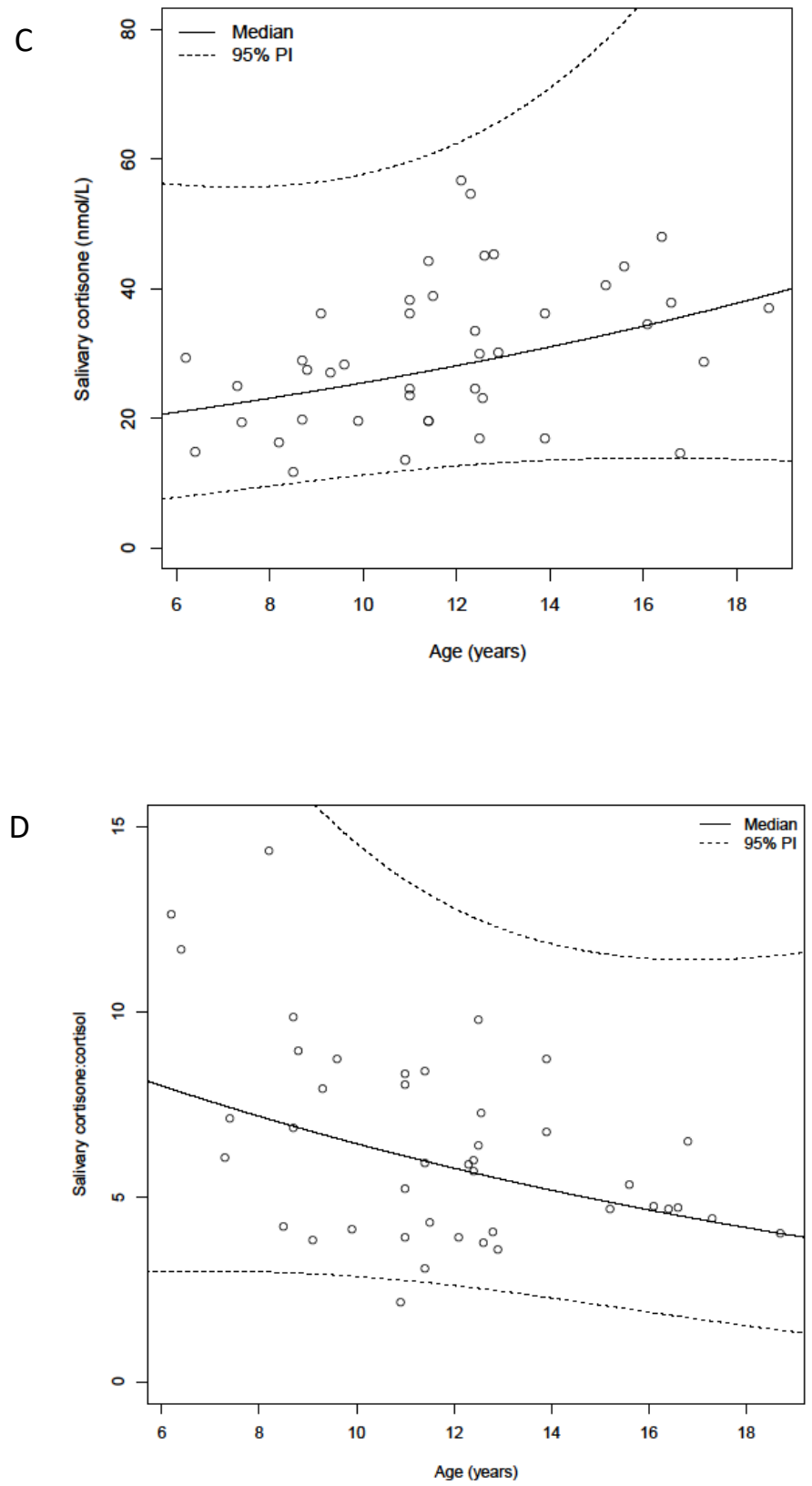